\title{
Deletion of the dominant autoantigen in NZB mice with autoimmune hemolytic anemia: effects on autoantibody and T-helper responses
}

\author{
Andrew M. Hall, ${ }^{1}$ Frank J. Ward,${ }^{1}$ Chia-Rui Shen, ${ }^{2}$ Cliff Rowe,${ }^{1}$ Laura Bowie, ${ }^{1}$ Anne Devine,${ }^{1}$ Stanislaw J. Urbaniak, ${ }^{1}$ \\ Christopher J. Elson, ${ }^{3}$ and Robert N. Barker ${ }^{1}$ \\ ${ }^{1}$ Department of Medicine and Therapeutics, University of Aberdeen, Aberdeen, United Kingdom; ${ }^{2}$ School of Medical Technology, Chang Gung University, \\ Tao-Yuan, Taiwan; and ${ }^{3}$ Department of Pathology and Microbiology, University of Bristol, Bristol, United Kingdom
}

\begin{abstract}
The mechanisms underlying apparently spontaneous autoimmune diseases, such as autoimmune hemolytic anemia (AIHA) in New Zealand Black (NZB) mice, are unknown. Here, we determine the contribution of the dominant red blood cell (RBC) autoantigen, the anion exchanger protein Band 3, to the development of NZB autoimmune responses. The approach was to prevent Band 3 expression in NZB mice by disrupting the $A E 1$ gene. $A E 1^{-1-}$ NZB mice produced RBC autoantibodies at the same levels as the wild-type
\end{abstract}

strain, but they differed in recognizing antigens that correspond to glycophorins, rather than Band 3. Splenic T-helper (Th) cells from wild-type NZB mice proliferated strongly against multiple Band 3 peptides, particularly the dominant epitope within aa861-874. This helper response was severely attenuated in $\mathrm{AE}^{-/-}$ animals, leaving only weak proliferation to peptide aa861-874. The results demonstrate that the defect in self-tolerance in NZB AIHA is directed to the RBC type, and is not specific for, or dependent on, Band
3. However, the predisposition to RBC autoimmunity may be focused onto Band 3 by weak Th cell cross-reactivity between the helper dominant epitope and an exogenous antigen. The redundancy of the major autoantigen illustrates the requirement for specific therapy to induce dominant forms of tolerance, such as T-cell regulation. (Blood. 2007;110: 4511-4517)

으 2007 by The American Society of Hematology

\section{Introduction}

The underlying mechanisms that drive damaging responses in autoimmune disease are poorly understood. Valuable insights have been obtained from the study of murine models. For example, genetic analyses have shown that different loci govern susceptibility to autoimmunity and determine which organs are affected, ${ }^{1}$ while functional studies have identified particular autoantigens and highlighted the role of T-cell subsets in pathogenesis. ${ }^{2}$ However, the stimuli that initiate and perpetuate apparently spontaneous autoaggression remain the subject of controversy.

Autoimmune hemolytic anemia (AIHA) is a classic example of an antibody-mediated autoimmune disease, and provides an opportunity to study the development of specific pathogenic responses, since the major human ${ }^{3-5}$ and murine ${ }^{6,7}$ autoantigens have been identified. Efforts to identify possible mechanisms of autoaggression often focus on spontaneous AIHA in the New Zealand Black (NZB) strain of mouse. The mice develop signs of anemia by 5 to 6 months of age due to the production autoantibodies that bind red blood cells (RBCs) and cause erythrophagocytosis. ${ }^{8}$ Band 3, the $\mathrm{RBC}$ anion exchanger protein (AE1), is the dominant autoantigen. ${ }^{6,7,9,10}$ Eluted autoantibodies exclusively immunoprecipitate Band 3 from murine RBCs, ${ }^{6}$ and 4 of 5 IgG anti-RBC monoclonal autoantibodies (mAbs) derived from NZB mice react with this antigen. ${ }^{9,10}$ The autoantibody response is helper dependent ${ }^{11,12}$ and Band 3 is also the major target for autoreactive T-helper (Th) cells. ${ }^{7,13-16}$ Splenic CD4 ${ }^{+}$T cells from NZB mice, but not H-2matched healthy strains, proliferate in vitro in response to Band $3,7,13$ and initial epitope mapping studies identified a number of

Submitted June 8, 2007; accepted August 22, 2007. Prepublished online as Blood First Edition paper, September 4, 2007; DOI 10.1182/blood-200706-094383.

An Inside Blood analysis of this article appears at the front of this issue. stimulatory peptides, of which aa861-874 was consistently the most potent. ${ }^{14}$ We have now identified the MHC binding and T-cell receptor (TCR) contact residues within Band 3 aa861-874, ${ }^{15}$ and confirmed that this epitope is dominant in vivo, since the administration of peptides containing the core sequence modulated autoantibody production and disease in NZB mice. ${ }^{16}$

There are a number of hypotheses put forward to explain the development of autoimmune diseases such as AIHA. ${ }^{17}$ One widely held belief is that disease is triggered by changes in the presentation of autoantigens from the target cells in such a way as to overcome self-tolerance. For example, infection or inflammation may activate antigen presenting cells (APCs) via innate pathways, ${ }^{18}$ leading to more effective autoantigen presentation to $\mathrm{T}$ cells, or may skew processing pathways within APCs to generate self-peptides that are less subject to central tolerance. ${ }^{19}$ A second explanation for autoaggression is that the damaging responses are a manifestation of polyclonal activators such as superantigens or mitogens, or of a generalized failure to control lymphoproliferation. ${ }^{20}$ Another commonly held view is that pathogenic autoimmunity is driven by antigens in the environment, particularly microbial proteins, which cross-react with the target autoantigen. ${ }^{18,19,21}$ This contention is supported by the induction of murine models, such as experimental autoimmune encephalomyelitis (EAE), ${ }^{22}$ by immunization with foreign homologues of self-proteins, but there is little evidence for crossreactivity or mimicry as a cause of apparently spontaneous autoimmune disease. ${ }^{17,23}$ marked "advertisement" in accordance with 18 USC section 1734. 


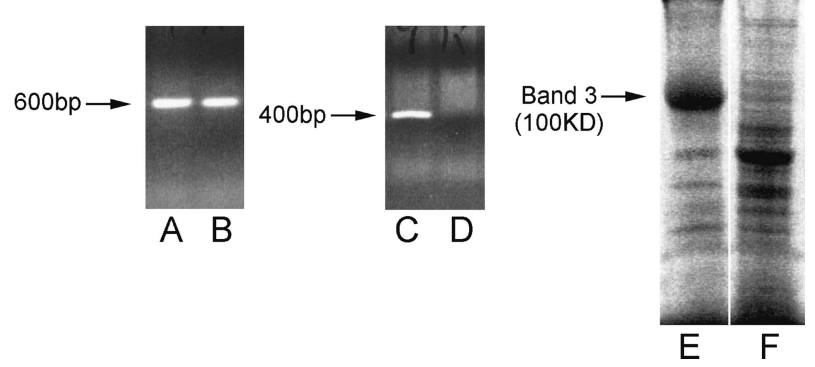

Figure 1. Lack of Band 3 expression in $A E^{-1-}$ gene-disrupted NZB mice. Lanes A-D illustrate ethidium bromide staining of agarose gel-fractionated PCR products corresponding to the disrupted (A,B, 600 base pairs) or wild-type (C,D, 400 base pairs) $A E 1$ gene sequence using samples of genomic DNA from heterozygous $A E^{+/-}$ $(A, C)$ or homozygous $(B, D) A E^{-1-}$ NZB mice. Lanes $E$ and $F$ illustrate SDS-PAGE analysis of wild-type $(E)$ or $A E^{-1-}(F)$ murine $R B C$ membrane preparations stained for protein with Coomassie Blue. Each track was loaded with approximately $20 \mu \mathrm{g}$ protein. The position of Band 3, with an apparent molecular mass of $100 \mathrm{kDa}$, is indicated $(\rightarrow)$.

One approach to testing these hypotheses is to determine the contribution of the dominant autoantigen to the induction of disease. Expression of any particular autoantigen in vivo may be unnecessary if disease is driven by aberrant immune processing and presentation of the target cell type, or by polyclonal activation, but would be required if the response were initiated by specific cross-reactivity with an environmental agent. The aim of the current work was therefore to test whether the development of AIHA in NZB mice is dependent on the expression of Band 3, or whether the spontaneous autoimmune response can switch to other RBC antigens. These questions were addressed by disrupting the $A E 1$ gene to prevent Band 3 expression, and then comparing autoantibody and $\mathrm{Th}$ responses in wild-type and $A E 1^{-1-} \mathrm{NZB}$ mice.

\section{Materials and methods}

\section{Mice}

NZB and healthy control BALB/c mice were maintained under specific pathogen-free conditions in isolators in the animal facilities at the universities of Bristol and Aberdeen. All work was carried out under United Kingdom Home Office Project License approval.

Peters et $\mathrm{al}^{24}$ provided $129 / \mathrm{SV}-\mathrm{C} 57 \mathrm{BL} / 6 \mathrm{~J}$ strain mice that lack the RBC anion exchanger Band 3 (129/SV-C57BL/6J.AE1 ${ }^{-1-}$ ) due to targeted disruption of the $A E 1$ gene. Failure to express Band 3 prevents the formation of important structural links with the internal membrane skeleton, and results in the production of fragile RBCs of abnormal morphology. ${ }^{24}$ However, mice homozygous for the null mutation can survive to adulthood, despite failure to express both RBC Band 3 and the related isoform of the protein from the kidney. ${ }^{24}$ Heterozygous $A E^{+/-}$mice have a normal phenotype and were used for a breeding program to back-crossing the mutant allele onto the NZB strain. Heterozygous $\left(A E 1^{+/-}\right)$offspring were identified by extracting genomic DNA from tail samples (Nucleon ${ }^{\mathrm{HT}}$ kit; Scotlab, Glasgow, United Kingdom) and using PCR to amplify a fragment specific for the mutant allele. ${ }^{24}$ After at least 20 successive cycles of back-crossing, heterozygotes were then intercrossed to generate offspring that include homozygous $A E 1^{-1-}$ NZB mice lacking Band 3. These were selected by demonstration of the mutant, but not wild-type, allele by polymerase chain reaction (PCR) and by the absence of Band 3 after sodium dodecyl sulfate-polyacrylamide gel electrophoresis (SDS-PAGE) analysis of RBC membranes (Figure 1).

\section{RBC membrane preparations}

Erythrocyte ghosts were prepared by hypotonic lysis of washed RBCs. ${ }^{3,6}$ The protein concentration of each sample was determined by the method of Bradford. ${ }^{25}$

\section{Antigens and mitogen}

Murine RBC antigens were prepared and their purity assessed as described previously. ${ }^{7}$ Briefly, murine Band 3 was purified by anion exchange chromatography from healthy murine RBC membranes that had been stripped of peripheral proteins and detergent solubilized in $1 \% \mathrm{C}_{12} \mathrm{E}_{8}$ (Sigma, Poole, United Kingdom). Residual detergent was removed by cold acetone precipitation of the protein, which was dialyzed extensively against phosphate-buffered saline (PBS), $\mathrm{pH}$ 7.4, before addition to cultures at a final concentration of $10 \mu \mathrm{g} / \mathrm{mL}$. The mitogen Concanavalin A (Con A; Sigma), was used to stimulate positive control cultures at an optimal final concentration of $1 \mu \mathrm{g} / \mathrm{mL}$.

\section{Peptides}

A panel of 92 15-mer peptides with 5 or 6 amino acid overlaps, corresponding to the complete sequence of murine Band $3,{ }^{26}$ was synthesized by a multiple-peptide solid-phase method using fluorenylmethoxycarbonylpolyamide chemistry (Department of Biochemistry, University of Bristol, Bristol, United Kingdom). The purity of the peptides was checked by mass spectroscopy. Peptides were used to stimulate cultures at $20 \mu \mathrm{g} / \mathrm{mL}$, which has been found to be the optimal concentration in previous epitope mapping studies. ${ }^{14-16}$

\section{Immunization with Band 3 protein}

Groups of 6-week-old NZB mice were immunized at the base of the tail by subcutaneous injection of Band 3 protein $(100 \mu \mathrm{g} /$ mouse $)$ administered as an emulsion in complete Freund adjuvant (Sigma).

\section{Culture and stimulation of $\mathrm{T}$ cells}

As described elsewhere ${ }^{7,13-16}$ splenic $\mathrm{T}$ cells were obtained and cultured together in the presence of antigen, peptides, or mitogen in a humidified atmosphere of $5 \% \mathrm{CO}_{2}$ and $95 \%$ air at $37^{\circ} \mathrm{C}$. On day 5 of culture, proliferation was estimated from the incorporation of tritiated $\left({ }^{3} \mathrm{H}\right)$ thymidine (Amersham, Aylesbury, United Kingdom) in triplicate samples using a 1450 Microbeta Liquid Scintillation Counter (LKB Wallac, Turku, Finland). Results are presented as stimulation index (SI), representing the ratio of proliferation in stimulated cultures versus unstimulated control wells. As previously, an SI greater than 3 was interpreted as a significant positive response. $7,13-16,27$

\section{Flow cytometry}

The antibodies used were directly conjugated with either fluorescein isothiocyanate or phycoerythrin (anti-mouse CD4, CD8, and CD71) and were purchased from Pharmingen (Oxford, United Kingdom). Cell samples containing $10^{6}$ cells were labeled with antibodies at optimal concentrations in the dark for 30 minutes on ice and washed. A benchtop FACScan analyzer IV (Becton Dickinson, Cowley, United Kingdom) was used with a $488 \mathrm{~nm}$ argon ion laser to detect green and red fluorescence, dead cells were excluded from analysis by gating on forward and side light scatter properties, and the data were analyzed with MAC Cell Quest (Becton Dickinson).

\section{Measurement of IgG subclasses bound to RBCs}

The number of $\operatorname{IgG}$ molecules of each subclass bound to the surface of RBCs was determined using a quantitative and sensitive cellular enzymelinked immunosorbent assay (ELISA) based on a direct enzyme-linked antiglobulin test (DELAT) developed in our laboratories and described in detail elsewhere. ${ }^{7,16,28}$ Briefly, a $2 \%$ suspension of washed, glutaraldehydefixed RBCs was added to duplicate wells of round-bottomed microtiter plates and incubated with subclass-specific sheep anti-mouse IgG antibody (The Binding Site, Birmingham, United Kingdom). After a further incubation with alkaline-phosphatase-conjugated donkey antisheep antibody (Sigma), the RBCs were allowed to react with phosphatase substrate solution (p-nitrophenyl phosphate; Sigma). The supernatant was transferred into flat-bottomed microtiter plates and the optical density (OD) measured at $405 \mathrm{~nm}$ (Titertek Multiscan II; Labsystems, Basingstoke, United Kingdom). The number of molecules of each murine $\mathrm{IgG}$ subclass bound to 
the RBCs was calculated by interpolation from a standard curve generated by tanning normal RBCs with a known concentration of each purified IgG subclass $^{7,16,28}$ (Sigma).

\section{Elution of autoantibody from RBCs}

Autoantibody was eluted from washed RBCs by ether treatment. ${ }^{3,6}$ Eluates were stored at $-20^{\circ} \mathrm{C}$ and, after thawing, any precipitated material was removed by centrifugation.

\section{SDS-PAGE}

SDS-PAGE was performed in $10 \%$ or $12 \%$ polyacrylamide gels as described previously ${ }^{3,6,29,30}$ according to the method of Laemmli, ${ }^{31}$ using the Mini Protean II (Bio-Rad, Hemel Hempstead, United Kingdom). Apparent molecular masses were calculated from migration relative to standard protein markers (SDS-6H; Sigma). Where necessary, gels were developed with Coomassie Brilliant Blue or periodic acid-Schiff (PAS) stains, respectively, for protein- or glycoprotein-containing bands. ${ }^{32}$

\section{Immunoprecipitation of RBC antigens}

Biotin-labeled murine RBC autoantigens or rat antigens were immunoprecipitated. ${ }^{3,6,29,30}$ Briefly, washed RBCs from healthy mice were biotinylated and sensitized with antibody from sera or RBC eluates. After incubation with rabbit antiserum against murine $\mathrm{IgG} F \mathrm{Fc}$ (Nordic, Maidenhead, United Kingdom), the RBCs were lyzed in hypotonic buffer and the membranes dissolved in PBS containing 2\% Triton X100 (Sigma). Solubilized immune complexes were immobilized on sepharose-protein A beads (Sigma) and eluted into gel sample buffer for SDS-PAGE. Proteins separated by SDS-PAGE were transferred to nitrocellulose by Western blotting, ${ }^{33}$ using a semidry apparatus (Pharmacia-LKB, Uppsala, Sweden) with a continuous buffer system as described by the manufacturer. Biotin-labeled RBC proteins bound to nitrocellulose were detected by incubation with peroxidaseconjugated Extravidin (Sigma) and luminography.,3,6,29,30,34 Blots were soaked in a freshly prepared luminescent substrate solution, ${ }^{34}$ sandwiched between polythene sheets and exposed to film (Hyperfilm MP; Amersham) in an X-ray cassette without intensifying screens.

\section{Results}

\section{Generation of $A E 1^{-I-}$ NZB mice}

The first step toward determining whether NZB AIHA is dependent on the dominant autoantigen, Band 3, was to generate genetically modified $A E 1^{-1-}$ mice that lack this protein. A targeted disruption of the AEl gene was introduced into $129 / \mathrm{SV}-\mathrm{C} 57 \mathrm{BL} / 6 \mathrm{~J}$ mice, ${ }^{24}$ back-crossed onto the NZB strain for at least 20 generations, and the offspring intercrossed to produce $A E 1^{-1-} \mathrm{NZB}$ mice. It has previously been demonstrated that mice homozygous for the null gene express no detectable Band 3 transcript or protein by Northern or Western blotting, respectively. ${ }^{24} A E 1^{-1-}$ mice for the current study were selected from the litters of $A E 1^{+/-}$intercrosses using PCR of genomic DNA ${ }^{24}$ to identify the null mutation and the absence of wild-type $A E 1$ gene, and by the lack of Band 3 seen after SDS-PAGE fractionation of RBC membranes (Figure 1).

\section{Anti-RBC antibody responses in gene-disrupted $A E 1^{-1-}$ NZB mice that lack the dominant Band 3 autoantigen}

If the defect in self-tolerance in NZB AIHA is not Band 3-specific, then the autoimmune response in $A E 1^{-1-} \mathrm{NZB}$ mice will switch to other RBC targets. However, if NZB disease results from polyclonal B-cell activation, then Band 3 antibodies should be produced despite absence of the antigen. On the other hand, if the initiation of autoimmunity is dependent on Band 3, for example, because it cross-reacts with an environmental agent, then there should be no

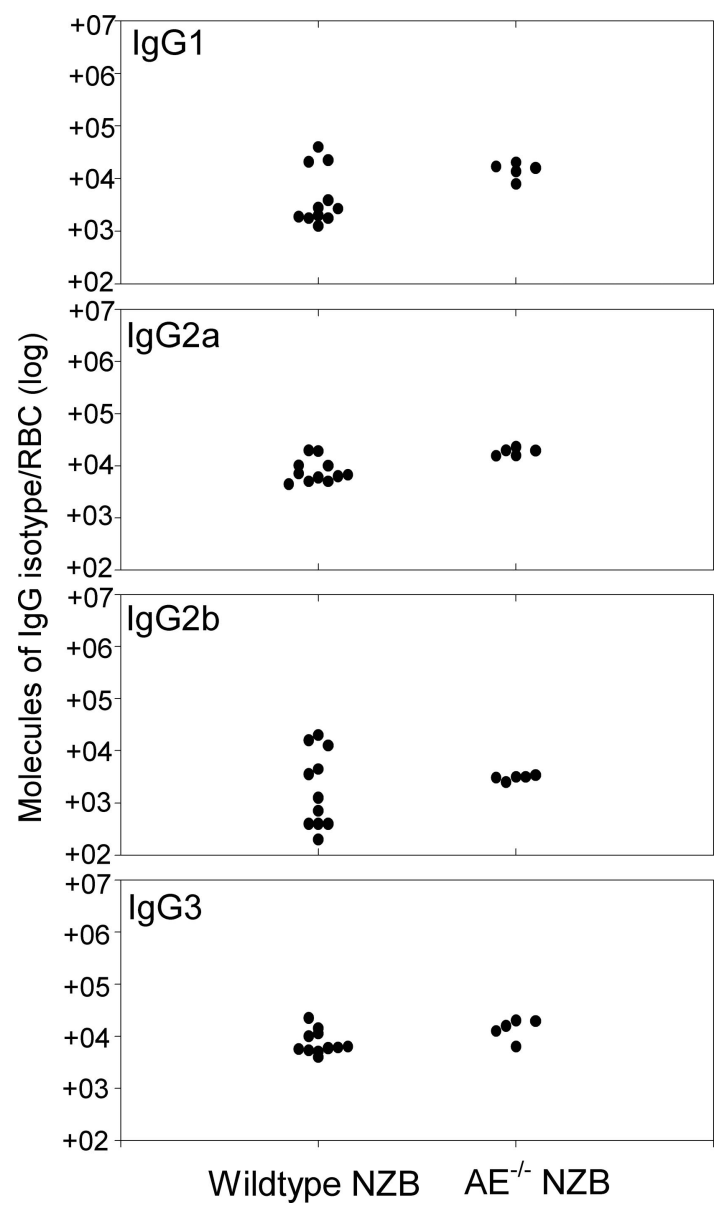

Figure 2. Autoantibody responses are equally strong in wild-type and $A E^{-1-}$ NZB mice. Comparison of number of IgG molecules of each isotype present on RBCs from individual wild-type $(n=16)$ or $A E^{-1-}(n=6)$ NZB mice aged 20 weeks. The IgG levels were measured by DELAT. The background levels on the RBCs of healthy control mice are less than 380 molecules $\operatorname{lgG}_{1}$, less than 250 molecules $\operatorname{lgG}_{2 a}$, less than 280 molecules $\lg _{2 b}$, and less than 500 molecules $\lg _{3}$.

disease in mice that lack the autoantigen. To test these predictions, we first asked whether anti-RBC antibodies are reduced or absent in NZB mice when the Band 3 autoantigen is not expressed, and then determined the specificity of any such antibodies.

The levels of RBC-bound IgG were compared in age-matched, wild-type, and $A E 1^{-1-} \mathrm{NZB}$ mice using a highly sensitive DELAT. ${ }^{28}$ It can be seen from Figure 2 that similar levels of all $4 \mathrm{IgG}$ isotypes were present on the surface of RBCs from both wild-type and $A E 1^{-1-} \mathrm{NZB}$ mice. Although the lack of Band 3 causes hemolysis through increased $\mathrm{RBC}$ fragility, ${ }^{24}$ autoantibody production in $A E 1^{-1-} \mathrm{NZB}$ mice is associated with more severe anemia than is seen in control nonautoimmune $A E 1^{-1-}$ mice. Mean hematocrit in $A E 1^{-1-} \mathrm{NZB}$ mice $(\mathrm{n}=6)$ was $11.0 \%$ plus or minus $2.5 \%$ standard deviation (SD), compared with $19.1 \%$ plus or minus $1.5 \% \mathrm{SD} A E 1^{-/-}$in animals $(\mathrm{n}=5)$ from the 129/SV-C57BL/6J strain.

The detection of high levels of RBC-bound $\operatorname{IgG}$ in $A E 1^{-1-} \mathrm{NZB}$ mice raises the question as to the specificity of this immunoglobulin. A related question is whether, in the absence of Band 3, the genetically modified animals make anti-Band 3 antibodies, which would be unable to bind their RBCs and therefore remain free in the serum. To address these issues, sera and antibodies eluted from the RBCs of $A E 1^{-1-}$ NZB mice were tested for the ability to immunoprecipitate labeled membrane antigens from healthy murine RBCs (Figure 3). Figure 3A illustrates the electrophoretic mobility of the major protein (i) and glycoprotein (ii) components 
A

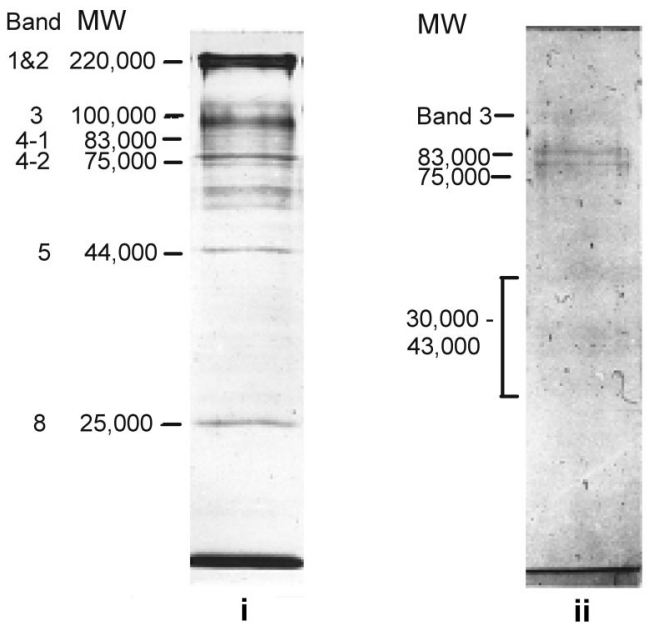

B

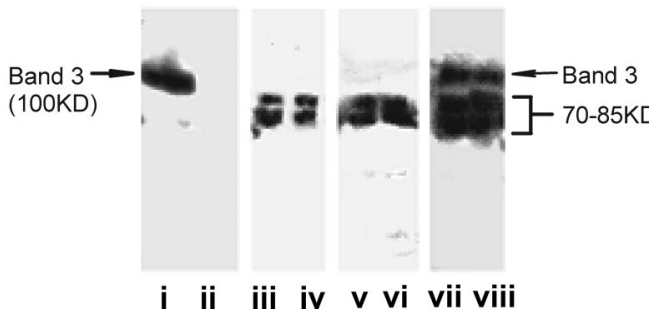

Figure 3. The spontaneous anti-RBC antibody response in $A E^{-I-}$ NZB mice targets antigens other than Band 3. (A) SDS-PAGE analysis of wild-type murine RBC membrane preparations stained for protein with Coomassie Blue (lane i), or for glycoprotein with PAS (lane ii). Each track was loaded with approximately $20 \mu \mathrm{g}$ protein. Major protein and glycophorin bands of the RBC membranes are labeled ${ }^{32}$ and the apparent molecular masses are indicated in $\mathrm{kDa}$. (B) Immunoprecipitation of biotin-labeled antigens from healthy murine RBCs. Immunoprecipitates were analyzed by SDS-PAGE, blotted onto nitrocellulose, and developed by luminography. Biotin-labeled RBCs were incubated with samples as follows: (i) positive control autoantibody-containing RBC eluate from wild-type NZB mice; (ii) negative control RBC eluate from healthy mice; (iii,iv) sera from individual $A E^{-1-}$ NZB mice; (v,vi) RBC eluates from individual $A E^{-1-}$ NZB mice; (vii,viii) control sera from individual $A E^{-/-}$ NZB mice immunized with Band 3 . The apparent molecular mass of the specific bands precipitated is indicated in $\mathrm{kDa}$. Lanes $\mathrm{i}$, ii, iii, vi, vii, and viii are from different blots. fractionated from wild-type murine RBC membranes. Figure 3B demonstrates that, as previously reported, positive control eluate from the wild-type NZB RBC immunoprecipitated one major protein species (i) corresponding in SDS-PAGE migration to Band 3. In contrast, no antibodies of this specificity were detected in sera (iii,iv) or RBC eluates (v,vi) from $A E 1^{-1-} \mathrm{NZB}$ mice. However, $\mathrm{IgG}$ in these samples instead precipitated a doublet of RBC proteins with apparent molecular masses of $70 \mathrm{kDa}$ to $85 \mathrm{kDa}$ in SDSPAGE. A comparison with stained RBC proteins and glycoproteins (Figure 3A) reveals that the precipitated bands correspond in mobility either to the internal membrane skeleton proteins Bands 4.1 and 4.2 , or to the highly glycosylated cell-surface glyocophorins. ${ }^{32}$ The diffuse nature of the precipitated bands is consistent with a high degree of glycosylation ${ }^{32}$ and, of the candidates, only the glycophorins would be available on intact RBCs for antibody binding. ${ }^{32}$ To demonstrate that $A E 1^{-1-}$ NZB mice retain the ability to make antibodies specific for Band 3, despite the absence of a spontaneous response, a control group of genetically modified animals was immunized with the purified protein as a foreign antigen. Figure 3B (vii,viii) shows that, in addition to the proteins of $70 \mathrm{kDa}$ to $85 \mathrm{kDa}$, sera from immunized $A E 1^{-1-}$ NZB mice also precipitated an RBC membrane component of $100 \mathrm{kDa}$, corresponding in SDS-PAGE migration to Band 3.

\section{Characterization of Th responses to Band 3 in wild-type NZB mice}

We have previously reported that splenic Th cells from NZB mice proliferate in vitro in response to Band 3 protein purified from $\mathrm{RBCs},{ }^{7,13}$ and to a dominant epitope contained within peptide aa861-874. ${ }^{14-16}$ It was now necessary to generate a complete helper epitope map of Band 3 in wild-type NZB mice with AIHA, for comparison with responses in gene-disrupted $A E 1^{-1-} \mathrm{NZB}$ mice that lack the autoantigen. A panel of 92 overlapping peptides corresponding to the complete sequence of Band 3 was screened for the ability to stimulate the in vitro proliferation of splenic T cells from NZB mice aged 20 weeks. Figure 4 summarizes the results from a series of up to 8 repeated experiments, and demonstrates that the $\mathrm{T}$ cells are capable of responding to a total of more than 20 peptides as judged by a stimulation index of 3 or
Figure 4. Proliferative responses of T cells from NZB mice to a panel of peptides spanning the sequence of the dominant murine RBC autoantigen Band 3 , and against the purified protein. Each bar represents the stimulation index of the peak response of T cells pooled from the spleens from individual mice aged 20 weeks. Each of the 92 peptides was tested on between 5 and 8 mice. The horizontal line indicates the minimum level of a positive response $(\mathrm{SI}>3)$.

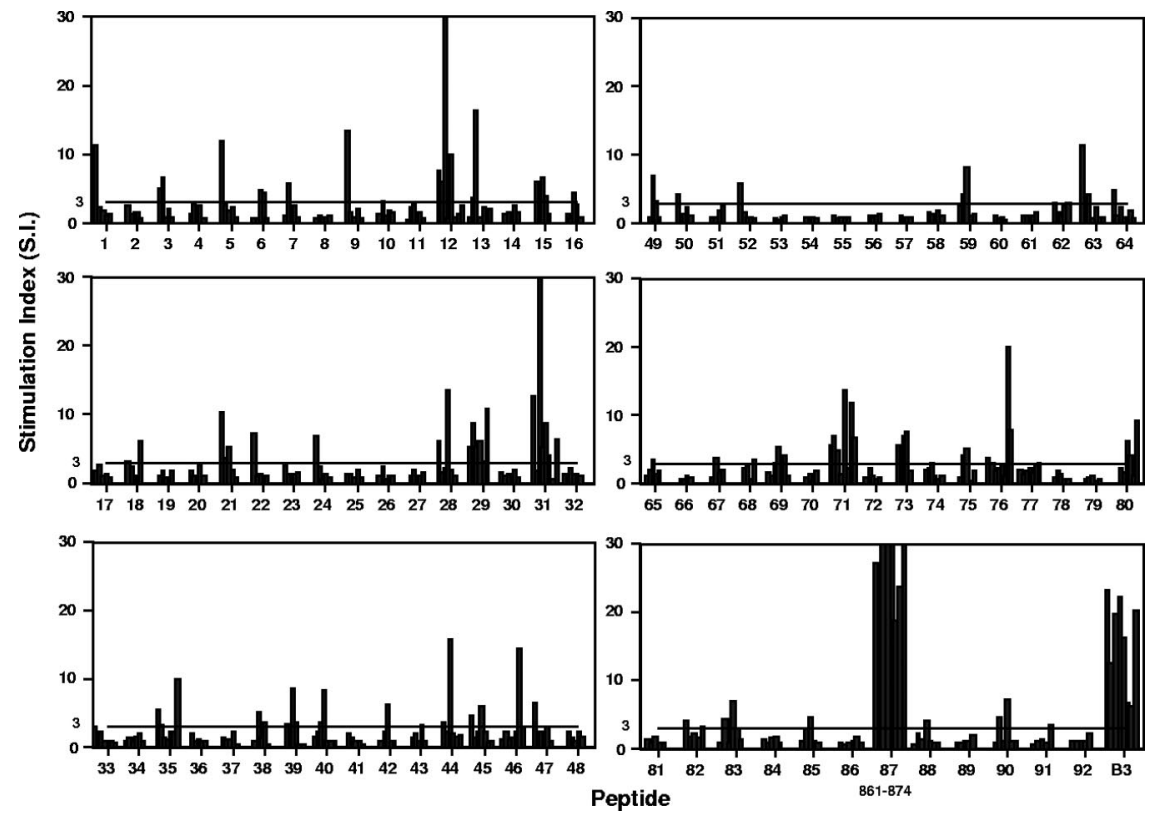


Figure 5. Proliferative responses of $T$ cells from $A E^{-1-}$ NZB mice to a panel of peptides spanning the sequence of murine RBC Band 3, and against the purified protein. Each bar represents the stimulation index of the peak response of T cells from individual mice aged 20 weeks. Each of the 92 peptides was tested on 6 mice. The horizontal line indicates the minimum level of a positive response $(\mathrm{SI}>3)$.
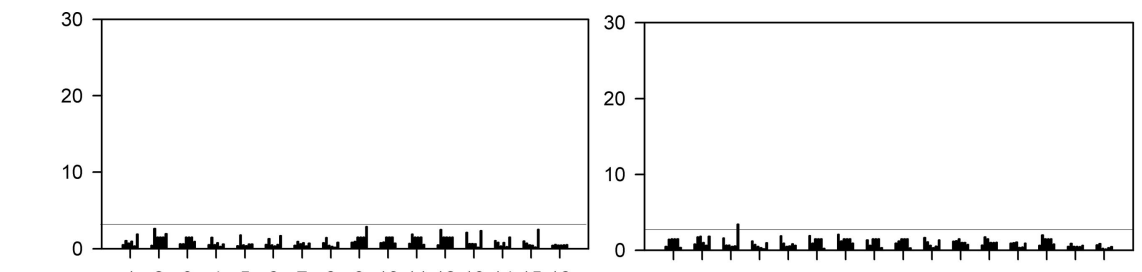

$\begin{array}{lllllllllll}1 & 2 & 3 & 4 & 5 & 6 & 7 & 8 & 9 & 1011 & 1213141516\end{array}$

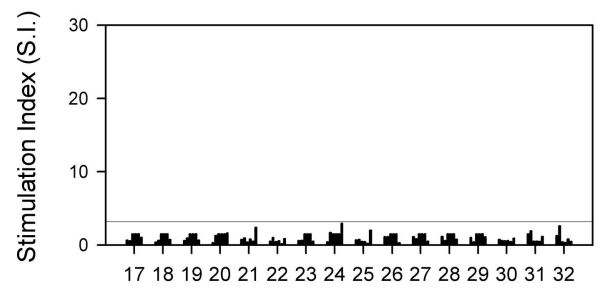

49505152535455565758596061626364
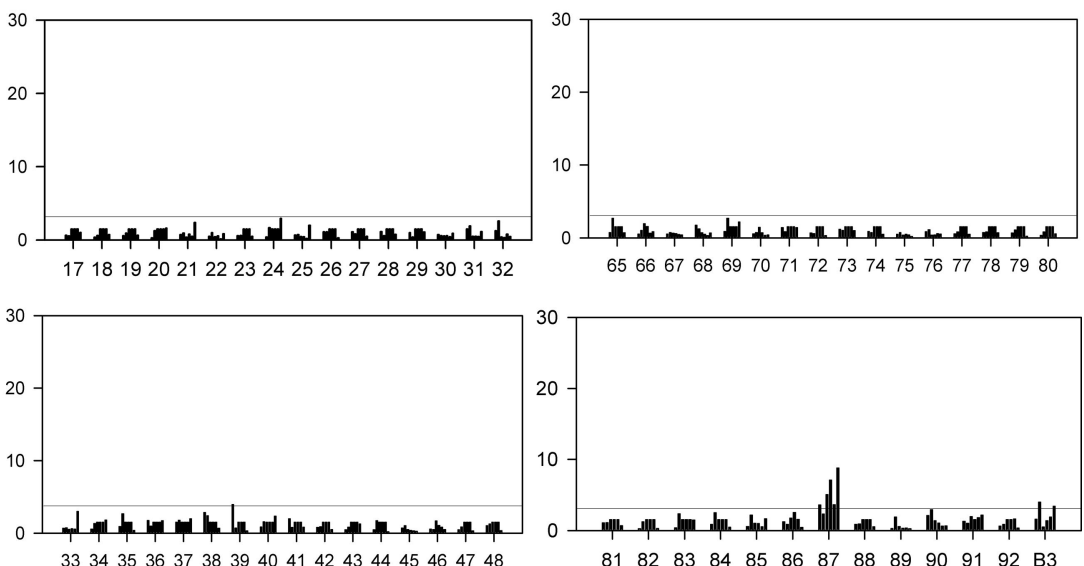

Peptide above. There were some differences in the profiles of stimulatory peptides between individual mice, but, as reported previously ${ }^{14-16}$ peptide 87 (aa861-874) invariably elicited strong responses. Purified Band 3 also potently stimulated proliferation in all experiments. Consistent with earlier studies, ${ }^{14-16}$ responses to Band 3 and peptides were predominantly mediated by $\mathrm{T}$ cells of the $\mathrm{CD} 4^{+}$ helper phenotype, as determined by subset depletion experiments, flow cytometric analysis, and the ability of antibodies against MHC class II molecules to block proliferation (results not shown).

\section{Characterization of Th responses to Band 3 in gene-disrupted $A E 1^{-1-}$ NZB mice that lack the autoantigen}

If autoaggressive Th cells in NZB mice are primed by polyclonal activators or cross-reactive environmental agents, rather than by Band 3, then there should be residual helper responses to Band 3 epitopes in the absence of the antigen in vivo. Gene-disrupted $A E 1^{-1-}$ NZB mice, matched in age with the wild-type NZB mice studied in Figure 4, were killed and their splenic Th cells tested for the ability to respond in vitro to the panel of Band 3 peptides and purified antigen (Figure 5). It can be seen that, in comparison with the results obtained using wild-type NZB mice, splenic Th cells from $A E 1^{-1-}$ animals were far less responsive to Band 3 protein and peptides. Thus, $A E 1^{-1-}$ Th cell proliferation against purified Band 3 and the subdominant peptides such as 12 (aa110-125) and 31 (aa301-317) was ablated or very weak. However, although responses to peptide 87 (aa861-874) were also markedly attenuated, significant reactivity to this dominant sequence was retained in all but one of the gene-disrupted animals.

It could be argued that the reduced proliferation against Band 3 epitopes in $A E 1^{-1-} \mathrm{NZB}$ mice is not specific, but due to general immunosuppression. However, the mitogen Con A was equally effective in stimulating splenic $\mathrm{T}$ cells from wild-type or genedisrupted NZB mice, and immunization of $A E 1^{-1-} \mathrm{NZB}$ mice with Band 3 restored in vitro responses to Band 3 and multiple peptides, including the dominant sequence aa861-874 (results not shown).

The finding that the dominant Band 3 peptide 87 (aa861-874) stimulates residual Th responses in vitro in $A E 1^{-1-} \mathrm{NZB}$ mice, albeit more weakly than in the wild-type strain, raises the possibility that the cells are primed in vivo by another antigen. Interrogation of the SwissProt database reveals considerable potential for such crossreactivity with, for example, multiple bacterial sequences identified that share all or some of the key MHC anchor and TCR contact residues with the autoreactive Th cell epitope in peptide $87 . .^{15}$

\section{Discussion}

This study of NZB mice genetically engineered to lack the dominant $\mathrm{RBC}$ autoantigen Band 3 has allowed dissection of the underlying mechanisms of spontaneous AIHA. The main result is that induction of NZB AIHA is not dependent on expression of the major autoantigen. In its absence, no detectable antibodies are made to Band 3, and the corresponding helper response is greatly attenuated, but, instead, high levels of autoantibodies with other RBC specificities are generated. The immune defect is therefore specific for the RBC type, rather than for the dominant autoantigen. Such plasticity of the autoaggressive response has implications for the specific treatment of AIHA and other autoimmune diseases.

The shift in the specificity of the autoantibody response in $A E^{-1-} \mathrm{NZB}$ mice indicates that the potential for autoaggression is not limited to Band 3, and is consistent with the view that a variety of antigens from the target cell may be processed and/or presented in a more immunogenic fashion to drive autoimmune disease..$^{2,17,19}$ For example, aberrant handling of RBCs by APCs would be expected to predispose to autoimmune responses against a range of potential autoantigens. ${ }^{17,19}$ Such a change may arise from activation of APCs, or recruitment of nonprofessional APC types, in response to innate immune signals. ${ }^{17-19}$ Immunoprecipitation experiments revealed that the major autoantigens bound by autoantibody in $A E^{-1-} \mathrm{NZB}$ mice correspond to the glycophorins, which are heavily glycosylated proteins expressed on the RBC surface. ${ }^{32}$ Glycophorins are important autoantigens in many other examples of AIHA, including 
disease in some human patients, $, 3,4,35,36 \mathrm{dogs},{ }^{30}$ and in experimental AIHA induced in mice by immunization with rat RBCs. ${ }^{29,37}$ Although studies of mAbs from NZB mice support the identification of Band 3 as the dominant RBC autoantigen, they also reveal the potential for autoantibodies with other, minor specificities to be present in NZB AIHA. ${ }^{9,10}$ Presumably such species, which are not detectable in the polyclonal response of wild-type NZB mice, expand greatly in the absence of Band 3. Autoantibody production in both wild-type and Band 3-deficient NZB mice was associated with anemia. Although the relative pathogenicity of the 2 antibody responses was not compared in detail, the levels bound to RBCs and the proportions of each IgG subclass were similar, and these factors are important determinants of hemolysis. ${ }^{13,16}$ The correspondence in the ratios of IgG isotypes also indicates that the Th subtype of the responses was unaffected by the switch in specificity.

The lack of anti-Band 3 antibody production in $A E^{-1-}$ NZB mice demonstrates that the response to this antigen in the wild-type strain is not a manifestation of polyclonal B-cell activation by, for example, superantigens. ${ }^{20}$ This confirms the results of a previous approach to determining the molecular mechanisms responsible for autoantibody production in NZB mice, based on characterization of anti-RBC mAbs derived from NZB splenocytes. ${ }^{9,10,38,39}$ The $\operatorname{IgV}_{\mathrm{H}}$ gene sequences of these mAbs, most of which are Band 3-specific, ${ }^{9,10}$ show evidence of somatic mutation, ${ }^{38,39}$ indicating that affinity maturation has occurred and that the autoantibody production is therefore antigen driven. The current work allows a further conclusion to be drawn: that the antigen responsible for driving the B-cell response in wild-type NZB mice is Band 3 itself.

Cross-reactivity or molecular mimicry between environmental and autoantigens has often been proposed to be a mechanism underlying apparently spontaneous autoimmune disease..$^{18,19,21,40}$ Such cross-reactivity may provide increased levels of particular antigenic sequences, or greater avidity of recognition, to overcome self-tolerance, and, in the case of mimicry by infectious agents, innate stimuli may enhance such immunogenicity. ${ }^{18}$ However, since AIHA in NZB mice is not dependent on expression of Band 3, it is now clear that the disease can develop without the need to invoke any cross-reactivity between environmental agents and this dominant RBC autoantigen. The possibility remains open that the same cross-reactive stimulus initiates responses to both Band 3 and glycophorin.

Given that Band 3 is not essential for NZB AIHA, and that the response can switch to other autoantigens, the question arises as to why Band 3 is the dominant target in wild-type NZB mice. One explanation is that dominance simply reflects the fact that there are more copies of Band 3 on the RBC surface than any other protein, ${ }^{32}$ and that the response in $A E^{-1-} \mathrm{NZB}$ mice switches to glycophorins as the next most abundant ${ }^{32}$ candidate antigens. However, analysis of Th responses in $A E^{-1-}$ NZB mice reveals a new possibility: that cross-reactivity with environmental agent(s) primes a weak Th response against the major Band 3 epitope, which, in the absence of the autoantigen, cannot spread to other determinants. Such epitope spreading, the recruitment of Th cells with further specificities for the initiating antigen, is a typical feature of autoimmune diseases, ${ }^{2,17,19}$ and is likely to be important in providing sufficient help to drive autoantibody production $^{41,42}$ and pathology. In the scenario suggested here, weak cross-reactivity with a single self-epitope acts to focus a tendency toward RBC autoimmunity onto a particular autoantigen, rather than representing a primary or sufficient cause of disease. Several examples of homology between the dominant RBC auto-epitope on Band 3 and bacterial sequences were identified, and it remains to be confirmed whether these, or any other environmental agents, are responsible for priming autoreactive Th cells in NZB mice.

Previous examples of murine genetic modification or mutations that prevent autoantigen expression have been described in other models of autoimmune disease. ${ }^{22}$ EAE can be induced by immunization with any one of several myelin proteins, and so lack of a particular target, such as myelin basic protein in mutant shiver mice, does not prevent disease induced by the other antigens. ${ }^{22}$ However, all such models are experimentally induced, and the current study of NZB AIHA is the first to demonstrate redundancy of a major autoantigen in a spontaneous autoimmune disease.

The ability of autoaggressive responses in NZB mice to switch to targets other than the dominant autoantigen is relevant to the development of specific immunotherapy for autoimmune conditions. Thus, even where a single dominant target for pathogenic responses can be clearly identified, reinstatement of tolerance to that autoantigen alone may not be an effective treatment. It will be essential to focus on the induction forms of active tolerance based on regulation, ${ }^{17,42}$ which may have bystander effects on other associated autoantigens, rather than simply the elimination or silencing of lymphocytes with one set of specificities.

\section{Acknowledgments}

The authors are very grateful to Dr Luanne L. Peters of the Jackson Laboratory, Bar Harbor, ME, for provision of gene-disrupted mice.

This work was funded by grants from the Wellcome Trust, United Kingdom (R.N.B.).

\section{Authorship}

Contribution: A.M.H., F.J.W., and C.-R.S. performed research, analyzed data, and wrote the paper; C.R., L.B., and A.D. collected and analyzed data; S.J.U. and C.J.E. designed research; R.N.B. designed research, collected and analyzed data, and wrote the paper.

A.M.H., F.J.W., and C.-R.S. contributed equally to this work.

Conflict-of-interest disclosure: The authors declare no competing financial interests.

Correspondence: R.N. Barker, Department of Medicine \& Therapeutics, Institute of Medical Sciences, University of Aberdeen, Foresterhill, Aberdeen AB25 2ZD, United Kingdom; e-mail: r.n.barker@abdn.ac.uk.

\section{References}

1. Vyse TJ, Todd JA. Genetic analysis of autoimmune disease. Cell. 1996;85:311-318.

2. Elson CJ, Barker RN. Helper T cells in antibodymediated, organ specific autoimmunity. Curr Opin Immunol. 2000;12:664-669.

3. Barker RN, Casswell K, Reid ME, Sokol RJ, El- son CJ. Identification of autoantigens in autoimmune haemolytic anaemia by a non-radioisotope immunoprecipitation method. Br J Haematol. 1992;82:126-132.

4. Leddy JP, Falany JL, Kissel GR, Passador ST, Rosenfeld SI. Erythrocyte membrane proteins reac- tive with human (warm reacting) anti-red cell autoantibodies. J Clin Invest. 1993;91:1672-1680.

5. Barker RN, Hall AM, Standen GR, Jones J, Elson CJ. Identification of T-cell epitopes on the Rhesus polypeptides in autoimmune hemolytic anemia. Blood. 1997:90:2701-2715. 
6. Barker RN, de Sa Oliveira GG, Elson CJ, Lydyard PM. Pathogenic autoantibodies in the NZB mouse are specific for Band 3 protein. Eur $\mathrm{J} \mathrm{Im}$ munol. 1993;23:1723-1726.

7. Perry FE, Barker RN, Mazza G, et al. Autoreactive $T$ cell specificity in autoimmune hemolytic anemia of the NZB mouse. Eur J Immunol. 1996; 26:136-141.

8. DeHeer DH, Edgington TS. Cellular events associated with the immunogenesis of anti-erythrocyte autoantibody responses of NZB mice. Transplant Rev. 1976;31:116-155.

9. de sa Oliveira GG, Izui S, Ravirajan CT, et al. Diverse antigen specificity of erythrocyte-reactive monoclonal autoantibodies from NZB mice. Clin Exp Immunol. 1996;105:313-320.

10. Diulio NA, Fairchild RL, Caulfield MJ. The antierythrocyte autoimmune response of NZB mice: identification of two distinct autoantigens. Immunology. 1997;91:246-251.

11. Chen S, Takeoka Y, Ansari AA, Boyd R, Klinman DM, Gershwin ME. The natural history of disease expression in CD4 and CD8 gene-deleted New Zealand Black (NZB) Mice. J Immunol. 1996;157: 2676-2684.

12. Oliveira GGS, Hutchings PR, Roitt IM, Lydyard PM. Production of erythrocyte autoantibodies in NZB mice is inhibited by CD4 antibodies. Clin Exp Immunol. 1994;96:297-304.

13. Shen C-R, Mazza G, Perry FE, et al. T-helper 1 dominated responses to erythrocyte Band 3 in NZB mice. Immunology. 1996;89:195-199.

14. Shen CR, Wraith DC, Elson CJ. Splenic but not thymic autoreactive T cells from New Zealand Black mice respond to a dominant erythrocyte Band 3 peptide. Immunology. 1999;96:595-599.

15. Shen CR, Ward FJ, Devine A, et al. Characterization of the dominant autoreactive T-cell epitope in spontaneous autoimmune haemolytic anaemia of the NZB mouse. J Autoimmun. 2002;18:149-157.

16. Shen C-R, Youssef A-R, Devine A, et al. Peptides containing a dominant T-cell epitope from red cell Band 3 have in vivo immunomodulatory properties in NZB mice with autoimmune hemolytic anemia. Blood. 2003;102:3800-3806.

17. Barker RN, Vickers MA, Ward FJ. Controlling autoimmunity: lessons from the study of red blood cells as model antigens. Immunol Lett. 2007;108: 20-26.

18. Ohashi PS, DeFranco AL. Making and breaking tolerance. Curr Opin Immunol. 2002;14:744-759.
19. Elson CJ, Barker RN, Thompson SJ, Williams NA. Immunologically ignorant autoreactive T cells, epitope spreading and repertoire limitation. Immunol Today. 1995;16:71-76.

20. Dziarski R. Autoimmunity: polyclonal activation or antigen induction? Immunol Today. 1988;9:340342.

21. Todd JA, Steinman L. The environment strikes back. Curr Opin Immunol. 1993;5:863-865.

22. Yoshizawa I, Bronson R, Dorf ME, AbromsonLeeman S. T-cell responses to myelin basic protein in normal and MBP-deficient mice. J Neuroimmunol. 1998;84:131-138.

23. Nicholson LB, Wraith DC. T-cell receptor degeneracy: the dog that did not bark: adaptation of the self-reactive T-cell response to limit autoimmune disease. Mol Immunol. 2004;40:997-1002.

24. Peters LL, Shivdasani RA, Liu SC, et al. Anion exchanger 1 (band 3 ) is required to prevent erythrocyte membrane surface loss but not to form the membrane skeleton. Cell. 1996;86:917-927.

25. Bradford MM. A rapid and sensitive method for the quantitation of microgram quantities of protein utilizing the principle of protein-dye binding. Anal Biochem. 1976;72:248-254

26. Kopito RR, Lodish HF. Primary structure and transmembrane orientation of the murine anion exchange protein. Nature. 1985;316:234-238.

27. Devereux G, Hall AM, Barker RN. Measurement of T-helper cytokines secreted by cord blood mononuclear cells in response to allergens. J Immunol Methods. 2000;234:13-22.

28. Mazza G, Day MJ, Barker RN, Corato A, Elson CJ. Quantitation of erythrocyte-bound IgG subclass autoantibodies in murine autoimmune haemolytic anaemia. Autoimmunity. 1996;23:245255.

29. Barker RN, Casswell KM, Elson CJ. Identification of murine erythrocyte autoantigens and crossreactive rat antigens. Immunology. 1993;78:568573.

30. Barker RN, Gruffydd-Jones TJ, Stokes CR, Elson CJ. Identification of autoantigens in canine autoimmune haemolytic anaemia. Clin Exp Immunol. 1991;85:33-40.

31. Laemmli UK. Cleavage of structural proteins during the assembly of the head of bacteriophage T4. Nature. 1970;227:680.

32. Barker RN. Electrophoretic analysis of erythrocyte membrane proteins and glycoproteins from different species. Comp Haematol Int. 1991;1: 155-160.

33. Burnette WN. "Western blotting": electrophoretic transfer of proteins from sodium dodecyl sulfatepolyacrylamide gels to unmodified nitrocellulose and radiographic detection with antibodies and radioiodinated protein A. Anal Biochem. 1981; 112:195-203.

34. Laing P, Parkar BA, Culbert EJ, Watt GJ, Elson CJ. Identification of rat erythrocyte antigens with a new non-radioactive immunoprecipitation technique. Scand J Immunol. 1987;25:613-62.

35. Garratty G, Arndt P, Domen R, et al. Severe autoimmune hemolytic anemia associated with IgM warm autoantibodies directed against determinants on or associated with glycophorin A. Vox Sang. 1997;72:124-130.

36. Johnson ST, McFarland JG, Kelly KJ, Casper JT, Gottschall JL. Transfusion support with RBCs from an Mk homozygote in a case of autoimmune hemolytic anemia following diphtheria-pertussistetanus vaccination. Transfusion. 2002;42:567571.

37. Barker RN, Shen C-R, Elson CJ. T-cell specificity in murine autoimmune haemolytic anaemia induced by rat red blood cells. Clin Exp Immunol. 2002;129:208-213.

38. Reininger L, Shibata T, Ozaki S, Shirai T, Jaton JC, Izui S. Variable region sequences of pathogenic anti-mouse red blood cell autoantibodies from autoimmune NZB mice. Eur J Immunol. 1990;20:771-777.

39. Scott BB, Sadigh S, Stow M, Mageed RA, Andrew EM, Maini RN. Anti-mouse red blood cell monoclonal antibodies use functionally rearranged genes from the $\mathrm{VH} \mathrm{J} 558$ family and are derived from the CD5- B-lymphocyte subpopulation. Immunology. 1993;79:568-573.

40. Barker RN, Elson CJ. Multiple self epitopes on the Rhesus polypeptides stimulate immunologically ignorant human T cells in vitro. Eur $\mathrm{J} \mathrm{Immu-}$ nol. 1994;24:1578-1582.

41. Stott L-M, Barker RN, Urbaniak SJ. Mapping alloreactive T cell epitopes on the Rhesus $D$ protein. Blood. 2000;96:4011-4019.

42. Hall AM, Ward FJ, Vickers MA, Stott LM, Urbaniak SJ, Barker RN. Interleukin-10-mediated regulatory T-cell responses to epitopes on a human red blood cell autoantigen. Blood. 2002;100: 4529-4536. 


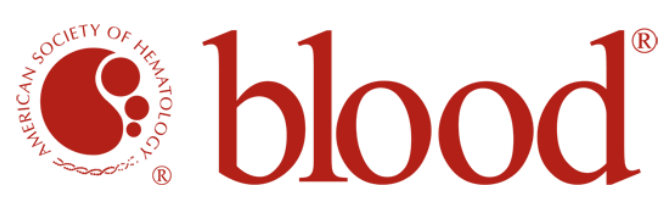

2007 110: 4511-4517

doi:10.1182/blood-2007-06-094383 originally published online September 4, 2007

\section{Deletion of the dominant autoantigen in NZB mice with autoimmune hemolytic anemia: effects on autoantibody and T-helper responses}

Andrew M. Hall, Frank J. Ward, Chia-Rui Shen, Cliff Rowe, Laura Bowie, Anne Devine, Stanislaw J. Urbaniak, Christopher J. Elson and Robert N. Barker

Updated information and services can be found at: http://www.bloodjournal.org/content/110/13/4511.full.html

Articles on similar topics can be found in the following Blood collections Free Research Articles (5175 articles)

Immunobiology and Immunotherapy (5627 articles)

Red Cells (1159 articles)

Information about reproducing this article in parts or in its entirety may be found online at: http://www.bloodjournal.org/site/misc/rights.xhtml\#repub_requests

Information about ordering reprints may be found online at:

http://www.bloodjournal.org/site/misc/rights.xhtml\#reprints

Information about subscriptions and ASH membership may be found online at:

http://www.bloodjournal.org/site/subscriptions/index.xhtml

Blood (print ISSN 0006-4971, online ISSN 1528-0020), is published weekly by the American Society of Hematology, 2021 L St, NW, Suite 900, Washington DC 20036.

Copyright 2011 by The American Society of Hematology; all rights reserved. 